\title{
Impact of bio-augmentation with Sphingomonas sp. strain TTNP3 in membrane bioreactors degrading nonylphenol
}

\author{
Magdalena Cirja • Gregor Hommes • \\ Pavel Ivashechkin • Jürgen Prell • Andreas Schäffer • \\ Philippe F. X. Corvini - Markus Lenz
}

Received: 14 April 2009/Revised: 18 May 2009/Accepted: 19 May 2009/Published online: 4 June 2009

(C) Springer-Verlag 2009

\begin{abstract}
This study evaluates the potential of bioaugmentation to improve the degradation of recalcitrant nonylphenol during the wastewater treatment in membrane bioreactors (MBR). One MBR containing activated sludge was bio-augmented using multistep inoculation with freeze dried Sphingomonas sp. strain TTNP3, whereas a second control reactor contained activated sludge solely. The ${ }^{14} \mathrm{C}$ labeled-nonylphenol isomer (4-[1-ethyl-1,3-dimethylpentyl] phenol) was applied as a single pulse. Bio-augmentation
\end{abstract}

Electronic supplementary material The online version of this article (doi:10.1007/s00253-009-2050-4) contains supplementary material, which is available to authorized users.

M. Cirja $\cdot$ A. Schäffer

Institute of Environmental Research-Environmental Biology

and Chemodynamics (BioV), RWTH Aachen University,

Worringerweg 1,

52074 Aachen, Germany

M. Cirja

Magdalena Vinken, Evonik Energy Services GmbH,

Rellinghauser Straße 1-11,

45128 Essen, Germany

P. Ivashechkin

VDEh-Betriebsforschungsinstitut $\mathrm{GmbH}$,

Sohnstrasse 65,

40237 Düsseldorf, Germany

J. Prell

Department of Molecular Microbiology, John Innes Center,

Norwich Research Park, Colney Norwich,

NR4 7UH, UK

G. Hommes $•$ P. F. X. Corvini $\cdot$ M. Lenz $(\bowtie)$

Institute for Ecopreneurship, School of Life Sciences, University

of Applied Sciences Northwestern Switzerland (FHNW),

Gründenstrasse 40,

4132 Muttenz, Switzerland

e-mail: markus.lenz@fhnw.ch resulted in an immediate increase of dissolved radioactivity in the effluent in comparison to the control reactor $(13 \%$ and $2 \%$ of initially applied radioactivity after 1 day, respectively). After 5 days of operation, the retentate of the bio-augmented reactor contained only $7 \%$ of the initial radioactivity in contrast to $50 \%$ in the control reactor. The radioactivity associated to the mixed liquor suspended solids, i.e., the suspension of biomass and other solids on the retentate side of the membrane, was mainly found as nonextractable residues that were increasingly formed during prolonged reactor operation, especially for the control MBR. HPLC-LSC and GC-MS ${ }^{\mathrm{n}}$ analyses revealed that the bioaugmented reactor produced more polar hydroquinone as main degradation intermediate, whereas the control reactor effluent contained a complex mixture of apolar compounds with shortened oxidized alkyl chains. Thus, the apparent differences in the behavior of nonylphenol between the reactors were due to the catabolism of nonylphenol conferred by bio-augmentation with Sphingomonas sp. strain TTNP3.

Keywords Bio-augmentation · Endocrine disruptors . Micropollutants $\cdot$ Biodegradation

\section{Introduction}

Micropollutants are found ubiquitously distributed in waters of both industrialized and remote environments. Due to the endocrine disrupting effect on aquatic organisms, nonylphenol (NP), a degradation product of NP polyethoxylates, has been widely studied over the last decade (Soares et al. 2008). Among the diverse mixture of occurring constitutional isomers of NP, 4-[1-ethyl-1,3-dimethylpentyl]phenol plays a special role, since it is resistant to biodegradation (presence of a quaternary $\alpha$-carbon), has one of the highest estrogenic 
potencies (MVLN cell lines) and is one of the most abundant isomers, accounting for approximately $20 \%$ of the total technical mixture (Cirja et al. 2006).

Sewage treatment plants suffer at times from biodegradation processes insufficient to ensure complete NP elimination, and the latter has been detected in many effluents (Cirja et al. 2008). One approach to increase this elimination efficiency is the addition of microorganisms capable of NP degradation, so called bio-augmentation. Bio-augmentation has been applied to improve the elimination of various xenobiotics (e.g., phenols, chloraniline, and aromatic hydrocarbons) from wastewater and wastewater treatment systems (e.g., paper mill, milk fat degradation, or olive mill wastewater; Boon et al. 2002; De Wildeman et al. 2004; Dhouib et al. 2006; Hailei et al. 2006; Loperena et al. 2006; McLaughlin et al. 2006; Olaniran et al. 2006).

However, bio-augmentation via simple addition of pure cell cultures is typically limited by washout and outcompetition of the inoculated culture by the endogenous microorganisms (El Fantroussi and Agathos 2005). Different strategies have been followed to supersede such drawbacks, such as, e.g., whole-cell immobilization in gels or beads (Fujii et al. 2003; Soares et al. 2006). Although promising in batch and short-term continuous lab experiments, the application of such immobilization approaches in full-scale wastewater treatment is still far from implementation: the successful immobilization of the microorganisms and the perpetuation of the degradation activity in long-term conditions under microbial competition have not been proven so far.

A promising alternative approach to immobilization in carrier materials may be the bio-augmentation in membrane bioreactors (MBR), which are of growing importance in full-scale (waste) water treatment applications. These reactors combine a biological treatment step with a filtration step on special membranes, resulting in almost complete retention of suspended solids, and, thus, biomass. Due to the increased sludge retention times (SRT) of the autochthonous microbial populations, an increased biodegradation of recalcitrant micropollutants can be an intrinsic feature of the MBR (Cirja et al. 2006). Although bio-augmentation in MBRs appears straightforward, competition for nutrients and inhibition by other substances contained in the wastewaters might still hamper its success.

This study investigates the bio-augmentation of MBRs with Sphingomonas sp. strain TTNP3, since it is one of the few bacteria able to degrade branched isomers of NP, which are recalcitrant to biodegradation. Sphingomonas sp. strain TTNP3 was chosen since it is able to degrade NP constitutively, no diauxic growth is observed in presence of other carbon sources, and it can degrade NP even at concentrations above $1 \mathrm{~g} / \mathrm{L}$ (Corvini et al. 2006b). In order to allow both identification of metabolites in complex matrices and assess the prevailing elimination mechanism (biodegradation versus sorption), 4-[1-ethyl-1,3-dimethylpentyl]phenol was applied as ${ }^{14} \mathrm{C}$-radiolabeled substance. Effects directly associated to bio-augmentation, i.e., the change of the metabolic pattern in the treated effluent, were investigated by single pulse spiking of NP (preventing adaptation of the autochthonous biomass) and multistep bio-augmentation of Sphingomonas (preventing a strong disturbance in MBR biocoenosis, since nutrients are less markedly depleted upon addition of exogeneous microorganisms). Furthermore, the long-term persistence of Sphingomonas within the MBR was demonstrated by polymerase chain reaction-denaturing gradient gel electrophoresis (PCR-DGGE).

\section{Materials and methods}

\section{Chemicals}

4-[1-Ethyl-1,3-dimethylpentyl]phenol was synthesized by means of a Friedel-Crafts alkylation of ${ }^{14} \mathrm{C}-\mathrm{U}-\mathrm{ring}$-labeled phenol with 3,5-dimethyl-3-heptanol (nonanol) in presence of $\mathrm{BF}_{3}$ as catalyst (Vinken et al. 2002). Chemical purity was $>98 \%$ and specific radioactivity was $1.492 \mathrm{MBq} / \mathrm{mg}$. Before spiking, the radiolabeled isomer was diluted with the nonradioactive form synthesized according to the same method.

\section{Source of biomass}

The MBR inoculum was sampled at a pilot scale MBR (wastewater treatment plant Soers, Aachen, Germany). Suspensions of resting cells of strain TTNP3 were prepared as described previously (Corvini et al. 2004b). For an easyto-apply solution allowing for possible long storage, the cells were lyophilized. The NP degradation activity of fresh cells and lyophilized cells was tested as described previously (Corvini et al. 2004b).

\section{Continuous MBR setup}

The lab-scale MBR designed for work under radioactive conditions was described in details previously (Cirja et al. 2006). Briefly, the system consisted of a 1.5-L glass cylinder hermetically closed with steel lids. The exhaust gas from the bioreactor vessel was bubbled sequentially through one flask containing $500 \mathrm{~mL}$ ethylene glycol (to trap volatile organic compounds) and two similar flasks containing $1 \mathrm{M} \mathrm{NaOH}$ solution (to trap $\mathrm{CO}_{2}$ ). Four microfiltration membrane plates were submerged in the activated sludge. 
Reactor operation and sampling

The lab-scale MBR was fed with synthetic wastewater adapted from DIN ISO 11733 (DEVL41), containing 700士 $2 \mathrm{mg} / \mathrm{L}$ chemical oxygen demand (COD), $40 \pm 5 \mathrm{mg} / \mathrm{L}$ total nitrogen (TN), and $5 \pm 0.5$ total phosphorus (TP). The medium was autoclaved at $120^{\circ} \mathrm{C}$ during $20 \mathrm{~min}$ and fed to the MBRs by means of a peristaltic pump. The reactors were operated with a SRT of 25 days at a hydraulic retention time (HRT) of $15 \mathrm{~h}$. The biomass concentration of the activated sludge was $10 \mathrm{~g} / \mathrm{L}$. The MBRs were inoculated with activated sludge and acclimated for 63 days to verify robustness of the membranes and stability of the process.

At the end of the stabilization period (day 64 ), ${ }^{14} \mathrm{C}-\mathrm{NP}$ (total of $3.8 \mathrm{MBq}$ ) was spiked at a concentration of $11.4 \mu \mathrm{mol} / \mathrm{L}$. Subsequently, a five-time inoculation strategy was applied. After $10 \mathrm{~min}$, the MBR was inoculated with lyophilized resting cells of strain TTNP3 (beforehand resuspended in distilled water) at a ratio of $100 \mathrm{mg} / \mathrm{L}$ of activated sludge. Similar inoculations were repeated after 24, 48, 72, and $96 \mathrm{~h}$. The control MBR was operated under the same conditions without addition of strain TTNP3.

Mixed liquor solid suspension (MLSS, $40 \mathrm{~mL}$ ) in the retentates was sampled at regular intervals and the total solid suspension content was determined. The effluent was monitored for water quality parameters, i.e. COD, TP, TN, and $\mathrm{NO}_{3}{ }^{-}$. At defined time intervals (e.g., before addition of Sphingomonas and $3 \mathrm{~h}$ after inoculation), samples of MLSS $(0.5 \mathrm{~mL})$, effluent $(1 \mathrm{~mL})$ and solutions for the trapping of stripped volatiles $(0.5 \mathrm{~mL})$, and stripped $\mathrm{CO}_{2}$ $(0.5 \mathrm{~mL})$ were sampled in triplicate and analyzed by means of liquid scintillation counter (LSC; Cirja et al. 2006).

Analytical techniques

Samples were analyzed using an HPLC device coupled to a radio detector (Cirja et al. 2006). For this, the sludge and effluent samples were extracted three times with ethyl acetate and extracts concentrated in a rotary evaporator to a final volume of $0.5 \mathrm{~mL}$. Identification of the metabolites was done after collection of the HPLC-separated fractions and subsequent analysis by GC-MS or LC-MS ${ }^{\mathrm{n}}$ (Cirja et al. 2006).

Non-extractable radioactivity was determined in ethyl acetate extracted pellets by combustion of $100 \mathrm{mg}$ pre-dried material and quantification of the resulting ${ }^{14} \mathrm{CO}_{2}$ by LSC (Cirja et al. 2006). GC-MS analyses were carried out as described previously (Vinken et al. 2002). COD, TP, TN, and $\mathrm{NO}^{3-}$ were determined spectrophotometrically using reactive test kits (Hanna Instruments, Italy). Temperature, $\mathrm{pH}$ value, and dissolved oxygen content were measured by electrodes in MLSS and effluent.
Microbial community analysis

Total genomic DNA was extracted from representative samples of MBR activated sludge taken before, during and after bio-augmentation ( 2 weeks after the radioactivity was exhausted in effluent). The DNA extraction, PCR reaction mixture, PCR conditions, and DGGE analysis are described in the Supplementary information. To verify the suitability of the extraction method, DNA from the pure culture of strain TTNP3 alone and from a mixture with activated sludge was performed as well.

\section{Results}

Effect of bio-augmentation on radioactivity balances

In the bio-augmented reactor, $60 \%$ of the initially applied radioactivity remained in the MLSS during the first day after initial bio-augmentation, whereas the radioactivity remained to more than $73 \%$ in the control reactor (Fig. 1). Simultaneously, a higher release of radioactivity with the effluent was observed in the bio-augmented reactor in comparison to the control (13\% and $2 \%$, respectively). During the next consecutive inoculations (days 2-5), this difference became more pronounced until the bioaugmented reactor contained only $7 \%$ in the MLSS in contrast to $50 \%$ in the control reactor (Fig. 1). From day 7 onward, sampling for radioactivity in the bio-augmented MBR was stopped, since neither the MLSS nor the effluent contained significant amounts of radioactivity. In contrast, the radioactivity present in the MLSS of the control reactor remained more than $10 \%$ of the initially applied radioactivity after 30 days of operation. Sorption to the components of the MBR accounted to $25 \%$ for the bio-augmented system and $30 \%$ for the control, respectively (radioactivity contained in the ethyl acetate extracts of the MLSS pellets determined by LSC). The removal of radioactivity through withdrawal of excess sludge was higher in the control in comparison to the bio-augmented reactor, due to the longer operational time (20\% and $9 \%$, respectively). Ultimate degradation of NP, i.e. the mineralized fraction $\left({ }^{14} \mathrm{C}-\mathrm{CO}_{2}\right)$, increased from $1 \%$ in the control experiment to $2.3 \%$ in the bio-augmented MBR. Volatiles were not found in significant amounts. In sum, the total recoveries were more than $96 \%$ of the initially spiked radioactivity for both MBRs.

In the first MLSS samples after NP spiking, most radioactivity was recovered in extractable form in both bio-augmented and control reactor (day 1, Fig. 2). Whereas the control reactor samples contained significant amounts of non-extractable residues in the sludge ( $>20 \%$, Fig. $2 b)$, the MLSS samples of the bio-augmented reactor showed higher amounts of dissolved radioactivity (32\%, Fig. 2a). 


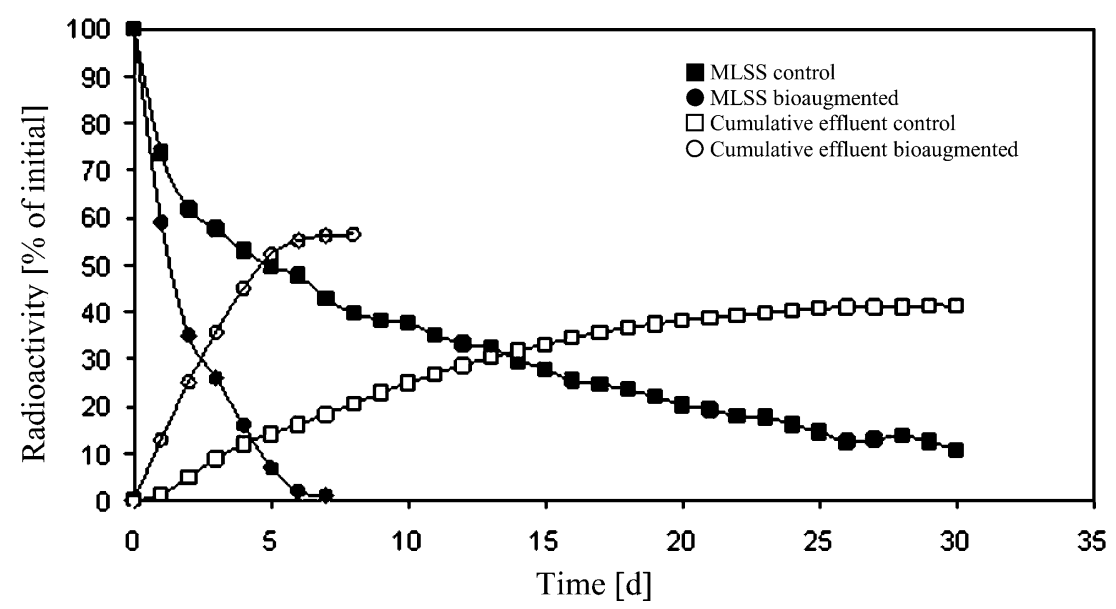

Fig. 1 Effect on radioactivity balance upon bio-augmentation. Radioactivity remaining within control and bio-augmented bioreactors in mixed liquor solid suspension $(M L S S)$ and cumulative radioactivity

During the time course of reactor operation, non-extractable residues were increasingly formed in the control reactor (>82\% Fig. 2b), whereas rather high amounts of dissolved and extractable species remained present in the MLSS of the bio-augmented reactor (Fig. 2a).

An immediate effect of bio-augmentation on formation of dissolved NP or NP metabolites in the permeate was observed shortly $(3 \mathrm{~h})$ after every inoculation with Sphingomonas (Fig. 3), e.g., a doubling of the dissolved radioactivity directly after the first inoculation (Fig. 3, II). After the last inoculation, radioactivity declined markedly, so that after 7 days nearly no radioactivity was released from the reactor via the effluent $(<1 \%$, Fig. 1$)$.

Effect of bio-augmentation on metabolic pattern during NP degradation

HPLC-LSC analysis revealed the NP degrading activity conferred by the application of Sphingomonas. In the bioaugmented reactors, only two major peaks in the HPLC chromatograms were observed, accounting to $45 \%$ (metab- release via the effluents. Note that $25 \%$ and $30 \%$ (bio-augmented and control, respectively) of the initial radioactivity was sorbed to the MBR materials olite at retention time of 4-5 min) and 55\% (parent NP, retention time of $22 \mathrm{~min}$ ) of the radioactivity detected in effluent extracts (Fig. 4, top). In contrast, the control reactor showed up to five metabolite peaks in the chromatogram (Fig. 4, bottom). The single peak areas contributed to $17 \%$, $12 \%, 13 \%, 44 \%, 11 \%$ (ascending retention time) of the total radioactivity injected. The parent NP was found in the effluent to minor amounts only (3\%). The sole metabolite in the bio-augmented reactor was identified by GC-MS analyses of the HPLC-collected fractions (between 3 and $5 \mathrm{~min}$ ) as hydroquinone (Fig. 4, top) (Corvini et al. 2006a), whereas the metabolites in the control reactor were identified by LC-MS ${ }^{\mathrm{n}}$ (Cirja et al. 2006) and consisted of compounds with shortened and oxidized alkyl chains (Fig. 4, bottom).

Persistence of Sphingomonas in bio-augmented continuously operated MBRs

The DNA-band pattern observed using PCR-DGGE analyses displayed additional DNA bands upon addition of Sphingo-
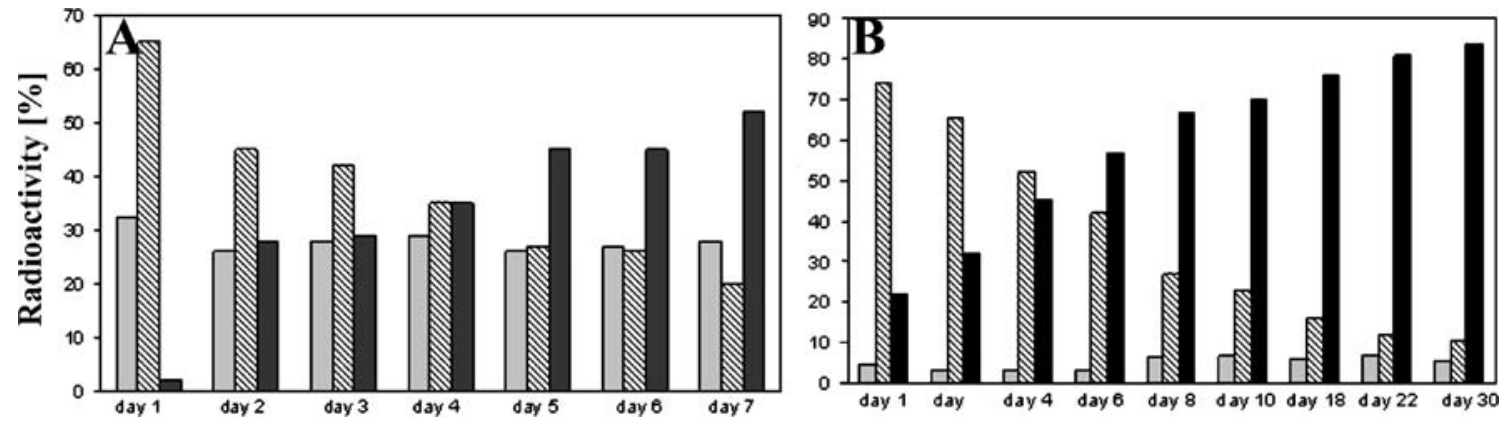

Fig. 2 Distribution of nonylphenol and metabolite thereof in mixed liquor solid suspension samples of the bio-augmented (a) and control (b) reactor: gray bars dissolved radioactivity, striped bars radioactivity extractable and black bars non-extractable from biomass 


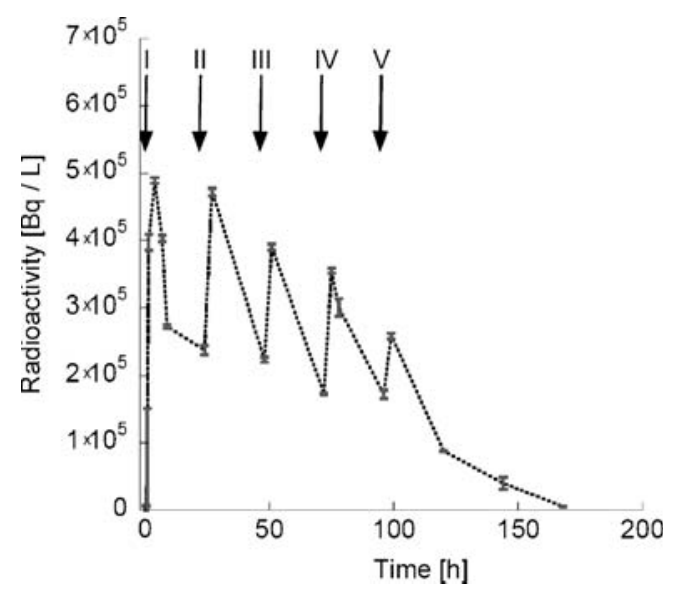

Fig. 3 Effect of bio-augmentation on formation of dissolved NP or NP metabolites in the permeate. Roman numbers mark the bio-augmentation pulses

monas sp. strain TTNP3 in comparison to the inoculum consisting of activated sludge (Fig. 1, Supplementary information). The bands appearing upon bio-augmentation persisted during NP (and metabolites thereof) presence in the effluent (days 1-5 after bio-augmentation) and at least 2 weeks after the radioactivity was exhausted in effluent (19-21 days after the bio-augmentation).

The quality of wastewater treatment by the two MBR systems was monitored by measuring $\mathrm{COD}, \mathrm{TN}, \mathrm{TP}, \mathrm{NO}_{3}{ }^{-}$in treated effluent in regular intervals (Table 1, Supplementary information). In both systems, COD was almost completely removed and nitrogen and phosphorus were assimilated by activated sludge microorganisms over the whole period (stabilization, NP spiking, bio-augmentation, and late phases). No remarkable disturbances concerning these parameters occurred in the system after inoculation of the MBR with Sphingomonas.

\section{Discussion}

Impact of bio-augmentation on the elimination of endocrine disruptors in MBRs

This study demonstrates that bio-augmentation in MBRs is a promising approach to increase elimination of endocrine disrupting substances that are otherwise insufficiently treated in conventional wastewater treatment. This was due to the fact that the membrane filtration step allowed retaining the added biomass and microbial degradation activity in long term, continuous reactor operation. Thus, using the MBR technology gave the possibility to overcome the commonly observed drawback of bioaugmentation, i.e., the washout of microorganisms.

Upon bio-augmentation with Sphingomonas sp. strain TTNP3, a degradation pattern significantly different from the non-bio-augmented system was observed. Whereas effluent radioactivity of the bio-augmented system consisted of NP and hydroquinone only (Fig. 4, top), the effluent of the control reactor contained a complex mixture of hardly degraded compounds with shortened and oxidized alkyl chains (Fig. 4, bottom). Since previous batch experiments showed hydroquinone being the central metabolite of NP degradation processing via type II ipso-substitution (Corvini et al. 2006b) and the shortened and the oxidized alkyl chains were previously reported in a non-bio-augmented MBR operated under similar conditions (Cirja et al. 2006), it was concluded that the change in metabolic pattern was a direct result of bio-augmentation with Sphingomonas sp. strain TTNP3.

The induced change in the metabolic pattern resulted in substantially different behavior of NP in the bio-augmented MBR. Due to its high $\log K_{\text {ow }}$ of 4.5, NP sorbs to solid particles and is poorly bio-available in aqueous environments,
Fig. 4 HPLC chromatograms of effluent extracts from bio-augmented (top) and control memrane bioreactor (bottom)

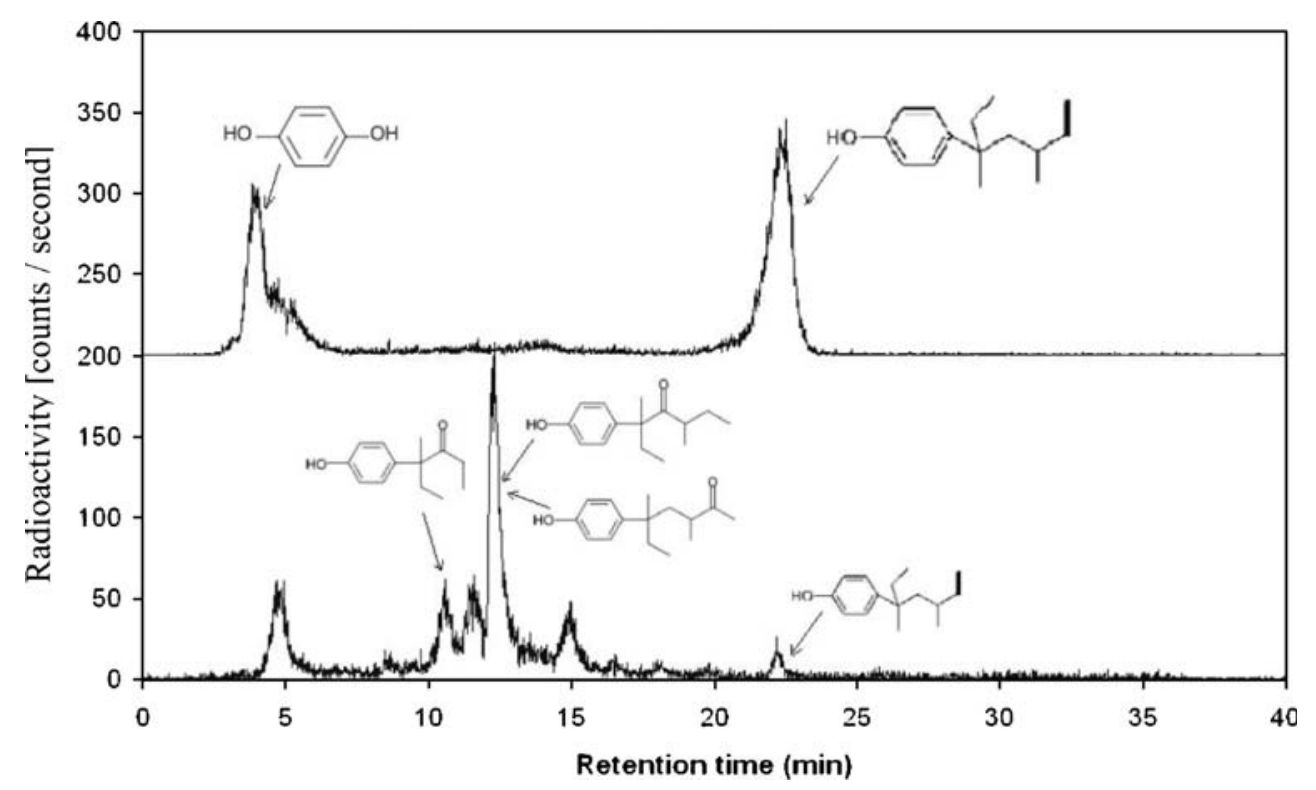


remaining recalcitrant to biodegradation during sewage treatment (Cirja et al. 2006). Hydroquinone, however, has a $\log K_{\text {ow }}$ of 0.59 only, thus, is expected to have a low sorption potential (Rogers 1996). In contrast, the majority of metabolites ( $80 \%$ of total injected radioactivity) in the control reactor probably bear a higher $\log K_{\text {ow }}$, since they elute later in the HPLC analysis (Fig. 4) and are structurally similar to the parent compound, NP. Consequently, the substantially higher radioactivity in the effluent of the bioaugmented MBR (Fig. 1), the higher share of water soluble radioactivity within the MLSS (Fig. 2), and the lower amount of radioactivity recovered from the reactor parts in contrast to the control reactor can be explained by different sorption behavior of the main metabolites.

Next to hydroquinone, NP contributed to the increased radioactivity in the effluent of the bio-augmented MBR. The fact that this highly sorptive compound was detected in the effluent can be due to the formation of biosurfactants, so called sphingans, a group of structurally related Extracellular Polymeric Substances (EPS) secreted by members of the genus Sphingomonas (Fialho et al. 2008). Such formation has been related with the increased solubility of other micropollutants (i.e., polyaromatic hydrocarbons), whereas the effect was most pronounced for the more hydrophobic compounds (Johnsen and Karlson 2004). Preliminary surface tension measurements demonstrated indeed that addition of strain TTNP3 to activated sludge clearly decreased the surface tension in comparison to activated sludge alone (Supplementary data). Therefore, a detailed investigation of sphingan formation and its influence on NP sorption during bioreactor operation should be undertaken in the future.

The degradation pathway of NP in strain TTNP3 has been studied and processes via ipso-substitution, ultimately leading to the formation of $\mathrm{CO}_{2}$ (Corvini et al. 2004b). In this study, however, ${ }^{14} \mathrm{CO}_{2}$ was only detected in minor amounts. This observation can be explained by the timescale necessary for complete degradation of nonylphenol isomers, which can be in frame of days to weeks (Corvini et al. 2004a, b). Thus, it can be reasonably assumed that NP can be more extensively removed under conditions that are favorable to hydroquinone degradation, e.g., under prolonged HRTs or partial recirculation of the permeate to the MBR system.

Beside the metabolic shift, the addition of Sphingomonas led to differences concerning the formation of nonextractable residues. Although in both reactors a formation of non-extractable residues was observed during the reactor operation (Fig. 2), this effect was more pronounced in the non-amended system, probably favored by longer contact time between NP, its metabolites and sludge matrix (7 versus 30 days, Fig. 2). In the non-bio-augmented system, entrapment of NP and its apolar metabolites in sludge particles may explain the formation of non-extractable residues (Kouloumbos et al. 2008a), while bound residues in the bio-augmented system might mainly result from the covalent binding of hydroquinone to the sludge matrix, similar to the reaction of hydroquinone with humic substances (Li et al. 2007).

Implications for practice

Attempts for bio-augmentation of wastewater and soil systems with strain TTNP3 and closely-related Sphingomonas cloacae as chitosan-encapsulated cells or as foam glass beads immobilized cells improved the degradation of NP in previous studies (Fujii et al. 2003; Soares et al. 2006); yet, these approaches might be prone to problems related to wash out of the microorganisms. In order to ensure sufficient degradation activity also under such wash out, bioaugmentation studies usually use an addition of high amounts of microorganisms to the medium to be decontaminated (e.g., 5\% volume used for groundwater bio-augmentation; De Wildeman et al. 2004). In the present study, bio-augmentation was done by multistep inoculation of small amounts of freeze-dried Sphingomonas cells directly into the retentate side of the MBR. This approach bears several crucial advantages for practical, large-scale applications: Firstly, the freeze-dried cultures can be prepared in advance and easily stored for elongated times upon application. Secondly, their addition has an immediate effect on the fate of endocrine disruptors in MBRs (Fig. 3); thus, cultures can be bio-augmented either in regular intervals or in case of acute contamination (e.g., peak loads by spillage during production processes). Since some NP degrading enzymes are dependent on NADPH/FAD (Kolvenbach et al. 2007) requiring active cells for regeneration of these cofactors, it is suggested that the lyophilized microorganisms were successfully rehydrated and active during bio-augmentation. Lastly, repeated inoculations of small amounts of the bacterium did not affect the wastewater treatment process (e.g., COD removal or nitrification efficiency, Supplementary data); yet, it was sufficient to maintain the NP degradation activity. In this regard, it was indicated by PCR-DGGE using a Sphingomonas-specific primer set that even 2 weeks after the last addition, strain TTNP3 was still present in the MBR. Since PCR-DGGE does not allow drawing conclusions on the viability of the strain, further studies should investigate how long NP degradation activity is sustained in order to determine necessary re-inoculation intervals for practice.

Whereas in the bio-augmented MBR only one metabolite with clearly different chemical properties compared to NP was formed, the complex mixtures of structurally similar metabolites in the control reactor raise considerable toxicological concerns. Due to the structural similarity to NP, the metabolites might still bear endocrine disrupting potential (Preuss et al. 2006), potentially in additional or 
even synergistic effects. In contrast, no endocrine disrupting effects are described for the exclusive metabolite, hydroquinone, found in the bio-augmented reactor. Therefore, it can be reasonably assumed that bio-augmentation led to a decreased endocrine disrupting potential of the effluent in the bio-augmented system. However, as various micropollutants contribute to the endocrine effect of wastewater treatment plant effluents in practice, this hypothesis needs to be verified under conditions using real wastewaters with fluctuations in their composition.

In case of NP, bio-augmentation had also a positive effect on the formation of extractable and non-extractable residues in comparison to the control reactor (Fig. 2). Such bound residues formation is not desired, since physically entrapped xenobiotics can be released from the sludge upon application in agriculture (Gevao et al. 2005; Kouloumbos et al. 2008b). Again, tests assessing the endocrine potential of sludge or soil mixture leachates should be undertaken when upscaling the process to full scale.

Acknowledgments We thank the European Commission for funding AQUAbase under the Human Resources and Mobility Activity within the sixth Framework Program under contract number MEST-CT-2004-505169.

\section{References}

Boon N, De Gelder L, Lievens H, Siciliano SD, Top EM, Verstraete W (2002) Bio-augmenting bioreactors for the continuous removal of 3-Chloroaniline by a slow release approach. Environ Sci Technol 36:4698-4704

Cirja M, Zühlke S, Ivashechkin P, Schäffer A, Corvini PFX (2006) Fate of a ${ }^{14} \mathrm{C}$-labeled nonylphenol isomer in a laboratory-scale membrane bioreactor. Environ Sci Technol 40:6131-6136

Cirja M, Ivashechkin P, Schäffer A, Corvini PFX (2008) Factors affecting the removal of organic micropollutants from wastewater in conventional treatment plants (CTP) and membrane bioreactors (MBR). Rev Environ Sci Biotechnol 7:61-78

Corvini PFX, Meesters RJW, Schäffer A, Schröder HF, Vinken R, Hollender J (2004a) Degradation of a nonylphenol single isomer by Sphingomonas sp. strain TTNP3 leads to a hydroxylationinduced migration product. Appl Environ Microbiol 70:68976900

Corvini PFX, Vinken R, Hommes G, Schmidt B, Dohmann M (2004b) Degradation of the radioactive and non-labelled branched 4(3', 5'-dimethyl 3'-heptyl)-phenol nonylphenol isomer by Sphingomonas TTNP3. Biodegradation 15:9-18

Corvini PFX, Hollender J, Ji R, Schumacher S, Prell J, Hommes G, Priefer U, Vinken R, Schäffer A (2006a) The degradation of $\alpha$-quaternary nonylphenol isomers by Sphingomonas $s p$. strain TTNP3 involves a type II ipso-substitution mechanism. Appl Microbiol Biotechnol 70:114-122

Corvini PFX, Schäffer A, Schlosser D (2006b) Microbial degradation of nonylphenol and other alkylphenols - Our evolving view. Appl Microbiol Biotechnol 72:223-243

De Wildeman S, Linthout G, Van Langenhove H, Verstraete W (2004) Complete lab-scale detoxification of groundwater containing 1, 2-dichloroethane. Appl Microbiol Biotechnol 63:609-612
Dhouib A, Ellouz M, Aloui F, Sayadi S (2006) Effect of bioaugmentation of activated sludge with white-rot fungi on olive mill wastewater detoxification. Lett Appl Microbiol 42:405-411

El Fantroussi S, Agathos SN (2005) Is bio-augmentation a feasible strategy for pollutant removal and site remediation? Curr Opin Microbiol 8:268-275

Fialho AM, Moreira LM, Granja AT, Popescu AO, Hoffmann K, Sá-Correia I (2008) Occurrence, production, and applications of gellan: Current state and perspectives. Appl Microbiol Biotechnol 79:889-900

Fujii K, Yamamoto R, Tanaka T, Hirakawa T, Kikuchi S (2003) Potential of a new biotreatment: Sphingomonas cloacae S-3 T degrades nonylphenol in industrial wastewater. J Ind Microbiol Biotechnol 30:531-535

Gevao B, Jones KC, Semple KT (2005) Formation and release of nonextractable ${ }^{14} \mathrm{C}$-Dicamba residues in soil under sterile and nonsterile regimes. Environ Pollut 133:17-24

Hailei W, Guosheng L, Ping L, Feng P (2006) The effect of bioaugmentation on the performance of sequencing batch reactor and sludge characteristics in the treatment process of papermaking wastewater. Bioprocess Biosyst Eng 29:283-289

Johnsen AR, Karlson U (2004) Evaluation of bacterial strategies to promote the bioavailability of polycyclic aromatic hydrocarbons. Appl Microbiol Biotechnol 63:452-459

Kolvenbach B, Schlaich N, Raoui Z, Prell J, Zühlke S, Schäffer A, Guengerich FP, Corvini PFX (2007) Degradation pathway of bisphenol A: Does ipso substitution apply to phenols containing a quaternary $\alpha$-carbon structure in the para position? Appl Environ Microbiol 73:4776-4784

Kouloumbos VN, Schäffer A, Corvini PFX (2008a) Impact of sewage sludge conditioning and dewatering on the fate of nonylphenol in sludge-amended soils. Water Res 42:3941-3951

Kouloumbos VN, SchaÌffer A, Corvini PFX (2008b) The role of sludge conditioning and dewatering in the fate of nonylphenol in sludge-amended soils. Water Sci Technol 57:329-335

Li C, Ji R, Vinken R, Hommes G, Bertmer M, Schäffer A, Corvini PFX (2007) Role of dissolved humic acids in the biodegradation of a single isomer of nonylphenol by Sphingomonas $s p$. Chemosphere 68:2172-2180

Loperena L, Saravia V, Murro D, Ferrari MD, Lareo C (2006) Kinetic properties of a commercial and a native inoculum for aerobic milk fat degradation. Bioresour Technol 97:2160-2165

McLaughlin H, Farrell A, Quilty B (2006) Bio-augmentation of activated sludge with two Pseudomonas putida strains for the degradation of 4-chlorophenol. J Environ Sci Health Part A Toxic/Hazard Subst Environ Eng 41:763-777

Olaniran AO, Pillay D, Pillay B (2006) Biostimulation and bioaugmentation enhances aerobic biodegradation of dichloroethenes. Chemosphere 63:600-608

Preuss TG, Gehrhardt J, Schirmer K, Coors A, Rubach M, Russ A, Jones PD, Giesy JP, Ratte HT (2006) Nonylphenol isomers differ in estrogenic activity. Environ Sci Technol 40:5147-5153

Rogers HR (1996) Sources, behaviour and fate of organic contaminants during sewage treatment and in sewage sludges. Sci Total Environ 185:3-26

Soares A, Guieysse B, Jefferson B, Cartmell E, Lester JN (2008) Nonylphenol in the environment: A critical review on occurrence, fate, toxicity and treatment in wastewaters. Environ Int 34:10331049

Soares A, Murto M, Guieysse B, Mattiasson B (2006) Biodegradation of nonylphenol in a continuous bioreactor at low temperatures and effects on the microbial population. Appl Microbiol Biotechnol 69:597-606

Vinken R, Schmidt B, Schäffer A (2002) Synthesis of tertiary ${ }^{14} \mathrm{C}$ labelled nonylphenol isomers. J Label Compd Radiopharm 45:1253-1263 